\title{
ZU DEN URSACHEN DER AUSBREITUNG (VON OSTEN NACH WESTEN) DER GLINA-KULTUR
}

Cristian Schuster

\section{EINLEITUNG.}

I.1. Im jetzigen Forschungsstand sind fast alle Archäologen, die sich eingehender mit der Glina-Kultur beschäftigen, damit einverstanden, daß diese frühbronzezeitliche Erscheinung hauptsächlich die Walachei und Oltenien als Verbreitungsgebiet hat ${ }^{1}$. Die Glina-Gemeinschaften haben in der Walachei nur die mittleren und westlichen Gebiete ${ }^{2}$ in Besiedlung genommen Die östliche Grenze liegt an der Mostiştea ${ }^{3}$, so wie uns die Funde von Săruleşti, Măgureni und Grădiştea-Făurei ${ }^{4}$ beweisen. Deswegen hat die ältere Hypothese von Ion Nestor ${ }^{5}$, die später von Jan Machnik ${ }^{6}$ neu aufgegriffen wurde, dernach auch der Osten der Walachei und sogar der Süden der Moldau oder der ganze Raum bis zum südlichen Bug von den Glina-Stämmen bewohnt wurde, ihren Wert verloren.

I.2. Zum Kerngebiet der Glina-Kultur ${ }^{7}$ muß der zentrate Teil der Walachei, d.h. der untere Lauf des Argeş-Flußes und seine Nebengewäßser (Colentina, Dâmboviţa, Neajlov, Câlnău, Câlniştea, Glavacioc u.a.), gerechnet werden. Es stellt sich die Frage, ob die Frühglina-Gemeinschaften (der I. Stufe ${ }^{8}$ ), gleichzeit mit ihrer Niederlassung (?) in der Walachei, auch in das Burzenland (Südostsiebenbürgen) eingedrungen sind oder ob sich dieser Prozeß erst später abgespielt hat. Unabstreitbar ist aber, daß die Glina-Kultur eine wichtige Rolle in der Entstehung der Schneckenberg-Kultur hatte'. Diesbezüglich äußerte Alexandru Vulpe den Gedanken, daß die ersten Funde der zuletzt erwähnten Kultur (d.h. Schneckenberg A) eigentlich zu der Glina-Kultur gezähit werden müßen und die jüngeren Spuren (Schneckenberg B) einer anderen kulturellen Äußerung (Năeni-Schneckenberg) angehören ${ }^{10}$.

Diese Hypothese, obwohl äußerst interessant, findet zur Zeit, wegen ihren ungenügend gereiften Argumente, nur einen schwachen Anklang in der neusten Fachliteratur ${ }^{11}$ und nimmt daher eine Sonderstelle ein. So daß die "alte" Meinung bezüglich zweier, zwar durch starken kulturellen Einflüße gebundenen, selbständigen Erscheinungen, von denen die Glina I-Stufe älter als die Schneckenberg A-Stufe ist, weiher eine solide Position bewahrt.

\section{URSACHEN DER BEWEGUNG.}

II.1. Die Wurzeln der Ursechen, die zu einem nach Westen tiehen eines Teils der GlinaBevölkerung führten, sind schwer zu ergründen. Deshalb wird unser Versuch wahrscheinlich nur ungenügend Licht auf dieses Problem werfen. Meistens wird dieses Thema vermieden oder die

\footnotetext{
${ }^{1}$ Sieh Schuster (1994, 63-70 und Abb. 1; 1997, 83-84 und Abb. 6) mit älterer Literatur

2 Schuster 1994, 63-66 und ABb. 1/A.

${ }^{3}$ Schuster 1994, 65-66; Schuster 1997, 83.

4 Şerbarnescu, Trohani 1978, 34.

5 Nestor 1960, Abb. 9.

6 Machnik 1991, 11.

${ }^{7}$ Schuster 1994, 64-65.

Öber die Periodisierung der Glina-Kultur sieh neulich: Schuster 1997, 84-87.

9 Über die Verbindungen in der Frühbronzezeit zwischen der Walachei und Südostsiebenbürgen s. neulich 5chuster 1998, 25-35.

${ }^{10}$ Siehe u.a. Vulpe 1990, 105-111.

${ }^{11}$ Zs. Székely 1997, 36, 50.
} 
verschiedenen Autoren drücken ihre Unfähigkeit das Problem zu lösen aus ${ }^{12}$. Die archäologischen Spuren erweisen sich diesbezüglich spärlich und sehr möglich jrreführend. Trotz dessen sind sowohl äußere als auch innere Gründe der Bewegung ersichtlich. Weiter muß von der Feststellung, daß in der Frühbironzezeit, die Gemeinschaften in dem von uns diskutierten Raum, in ständiger Bewegung waren und dieses Herumstreifen verschiedene Formen annahm ${ }^{13}$, ausgegangen werden.

\section{II.2. Innere Beweggründe.}

\section{I1.2.1. Wirtschaftliche Ursachen.}

Das Glina-Wirtschaftsleben stützte sich auf die Viehzucht, den primitiven Ackerbau, die Jagd und den Fischfang und weniger auf die Gewerbe, den Transport und den Handel ${ }^{14}$. Selbverständlich spielte die Viehzucht die wichtigste Rolle ${ }^{15}$.

Die Analyse der Tierknochenreste zeugt davon, daß die meistgezüchteten Tiere der Bos Taurus, die Ovies Avies und Capra Hircus ${ }^{16}$ waren. Dlese in Herden lebende Tierarten hatten für ihre Nahrung ein großes Hinterland nötig. Ein Beweis diesbezüglich ist die Dichte der Siedlungen ${ }^{17}$ (kleine Dörfer, eigentlich Weiler), die nicht nur durch ein Bevölkerungswachsen, sondern hauptsächlich durch die wirtschaftliche Notwendigkeit der Nahrungssuche für die Tiere, zu erklären ist. Noch zu nennen sind der hohe Prozentsatz der Gefäßformen ${ }^{18}$, die für die Aufbewahrung der Milch und Milchprodukte verwendet waren, und nicht zuletzt die Anwesenheit der Hunde ${ }^{19}$, die nicht nur für das Bewachen der Dörfer sondern auch der Herden und für die Jagd nötig waren.

Neben der Viehzucht ist auch die Jagd nicht zu vergeßen, die für die Bereicherung der Nahrung sorgte. Obwohl unter den Waffen ${ }^{20}$, hauptsächlich Steingeräte, die Pfeilspitzen sehr selten entdeckt wurdeı (Crivăt ${ }^{21}$, Varlaam ${ }^{22}$, Brane ${ }^{23}$ ), gibt es genügend Messer, Schaber, Klingen, die bei der Jag̣, aber auch beim Schlachten oder Abhäuten der Tiere benütut wurden.

Das Wirtschaftsleben prägt die innere Entwicklung der Gemeinschaften. Diese haben eine kleine Anzahl von Mitgliedern, wahrscheinlich 30-36 Personen ${ }^{24}$. Die Siedlungen bestehen aus einer geringen Zahl von Behausungen (Oberfächen- oder Bodenwohnungen) ${ }^{25}$, deren Aufbauweise sehr einfach war: ein Holzgerüst, Ruttengeflecht und Lehmbewurf. Diese Bautechnik zeugt von einer Bevölkerung, die die leicht und schnell errichtbaren Wohnhäuser bevorzugte, da sie in ständiger Bewegung war. Sie ließ sich nür für eine kurze Zeit an leinem Ort nieder, so wie uns auch die dünne Kulturschicht der Großzahl der Siedlungen erleuchten ${ }^{26}$. Deshalb legten sie wahrscheinlich nicht zu großen Wert auf Befestigungsanlagen, daher auch die kleine Anzahl der Wehrgraben ${ }^{27}$.

Möglich ist weiter, daß die Wirtschaftsart ınd implizitte die ständige Herumpendelung der Gemeinschaften zu einer Bestattungsäußerung führte, die nicht das Gründen von Nekropolen

\footnotetext{
12 Roman et all. 1992, 118.

${ }^{13}$ Schuster 1998a, 187-191.

${ }^{14}$ Schuster 1997, 135-146.

15 Schuster 1997, 135-137.

16 Haimovici 1997 (im Druck); Schuster 1997, 135.

${ }^{17}$ Schuster 1997, 29-33.

${ }^{18}$ Schuster 1997, 60-66: besonders die GefäBbormen 1-7.

19 Schuster 1997, 137.

20 Schuster 1997, 49-53.

21 Păunescu 1970, 204.

22 Schuster 1997, 51.

${ }^{23}$ Ulanici 1979, 27-28.

24 Schuster 1997, 46.

${ }^{25}$ Schuster 1997, 33-41; Schuster 1997a, 85-104.

${ }^{26}$ Sieh unter anderen: Schuster 1997, 29-33.

${ }^{27}$ Schuster 1997, 30-31.
} 
erlaubte ${ }^{28}$, denn, außer den Glina spätzeitlichen Grabhügel von Verbiţa (Glina IV-Stufe) ${ }^{29}$, sind keine sichereinstufbaren Grabstätten oder Gräber bekannt ${ }^{30}$.

\section{II.2.2. Der demographische Faktor.}

Wenn das Wirtschaftsleben die Hauptrolle einnimmt, muß auch dem demographischen Faktor die nötige Aufmerksamkeit geschenkt werden. Dieser kann aber nur im festen Zusammenhang mit der getriebenen Ökonomie verstanden werden. Hinzu sind desgleichen die natürlichen Bedingungen in denen die Glina-Gemeinschaften ihr Leben geführt haben, zu zähien. ES ist zu unterstreichen, daß insbesondere das Kerngebiet sehr stark bewaldet, war, so daß hauptsächlich nur die Nachbarzonen der Geiwässer für die Ansiedlung und als Nahrungsraum für die Tiere benützt wurden ${ }^{31}$.

Wie wir schon gezeigt haben, bestend 'eine Glina-Dorfgemeinschaft aus relativ wenig Mitglieder. Jefler Bevölkerungs-zuwachs, durch Geburt oder "Heirat" führte zu einer Belästigung des Gemeinschaftslebens (Nahrungsprobleme ?). Deswegen müßen wir nicht aussçhließen, daß die neuen Familien ausgestoßen wurden und den Kern einer neuen Gemeinschaft bildeten, so wie es auch in anderen Perioden der Fall war. Auch diese Seite des sozialen Lebens könnte ein Grund der Siedlungsdichte sein.

\section{II.3. Äußere Gründe.}

II.3.1. Dumitru Berciu äußerte in den ' 30 Jahren die Meinung, daß die Glina-Gemeinschaften nach Oltenien entlang der Donau aus Mitteleuropa vordrangen ${ }^{32}$.

Heute sind alle Archäologen, die sich mit dieser Kultur beschërtigen, damit einverstanden, daß der Weg eigentlich vom Osten nach Westen führte und nicht umgekehrt ${ }^{33}$.

II.3.2. Der jetzige Forsthungsstand zeigt, daß Spuren der Gline I-Stufe nicht in der Nähe der Donau zu finden waren. Erst in der klassischen Periode ( $d . h$. ihr erster Abschnitt = Glina II) sind Glina-Siedlungen nicht direkt am linken Stromufer, sondern an den Donau-Seen (z.B. Greaca, Cătălui) zu vermerken ${ }^{34}$. In ihrem Vormarsch nach Westen über den Alt (OIt) siedeln die Glina-Leute zwar Orte, die in der Nachbarschaft der Donau liegen, an ${ }^{35}$, aber diese sind gering in der Zahl. Die wichtigste Glina-Siedlung liegt in Westoltenien auf der Ostrovul Corbului-Donauinse $\left.\right|^{36}$.

Diese Seltenheit der Glina-Funde an der Donau sind entwecier das Ergebnis der lückenhaften Forschungen oder, eher, eine Äußerung der Tendenz, daß die Glina-Stämme die Donau vermieden haben. Wahrscheinlich waren die Lebensbedingungen ungeeignet und weiter erschwerte das aus dem Osten kommen der nicht Glina- Völkerschaften und die weiter nach Westen zogen, und dabei das Donautal benüttten und winhrscheinlich kontrollierten, das Glina-Niederlassenı am Donauiifer.

Interessant ist aber, wie die meistens Autoren hervorheben, daß eigentlich in der erwähnten Zeit und im genannten Raum (Karpaten-Donau-Moldau-Gebiet) auch von einer entgegengesetzten Strömung, d.h. vom Westen nach Osten, geredet werden mu $\beta^{37}$. Valentin Dergacev spricht sogar von

\footnotetext{
2 Schuster 1997, 47-48.

29 Berciu und Roman 1984, 15-21; Roman et all. 1992, 115-116.

30 Schuster 1997, 47-48.

"Schuster 1997, 29-30.

3 Berciu 1934, 11.

${ }_{33}$ Roman 1976, 26-42; Roman 1985, 116-122; Roman 1985a, 279-297; Schuster 1994, 63-70; Schuster $1997,83-84$.

${ }^{34}$ Schuster 1997, 185-187, 193.

35 Z.B. Corabia, Bezirk Olt: Berciu 1934, 12-13 und Karte mit den Glina-Funden aus Ottenien.

3 Roman 1996, 1-33 (in deutscher Sprache: 35-65) mit alterer Literatur.

7 Dergacev 1989, 135-136.
} 
einem "Migrationsstrom"38, der u.a. die Funde des Edineţ-Kulturtyps besser erklären könnte ${ }^{39}$. Unseres Erachtens sind selbstverständlich auch Bevölkerungsbewegungen in Betracht zu ziehen, aber diese müssen nicht übertrieben werden. Eine Mixtur von geringen "ethnischen" Elementen und hauptsächlich starke kulturelle Einflüße sind bei der Deutung der Ähnlichkelt des Glina- und Edinetabel auch des Schneckenberg B-Materials maßgebend. Dieser Einfluß erfolgte nicht entlang der Donau, sondern seine Spuren sind im muntenischen Hügel- und Vorkarpatengebiet und weiter im südöstlichen Raum Siebenbürgens und in der Moldau und leaten Endes am linken Ufer des mittleren Pruth Zu erkennen ${ }^{40}$.

Die Glina-Kultur ist zeitgleich mit der Bugeac-Gruppe der Grubengrabkultur. Die letzte Erscheinung nimmt Teile des Dnertr-Dnjepr-Binnenlandes, das Gebiet der Republik Moldawien und weiter westlich die der östliche Teil der Moldau, die rumănische Dobrudgcha und den Nordosten Bulgariens ein ${ }^{41}$.

Diesbezüglich stellt sich die Frage ob auch der ostliche Teil der Walachei zum Verbreitungsgebiet der Bugeac-Gruppe gehört hat. Auf der Karte (Kulturen der frühen Bronzezeit der pontisch-kaspischen und der Karpaten-Balkan-Region im 1. Viertel des 2. Jahrtausends v. Chr.) veröffentlicht von Larisa Bogataja und Igor Manzura ${ }^{42}$ und jene von Manzura und Sava ${ }^{43}$, eigentlich ein und dieselbe, wird auch das genannte Gebiet der Grubengrabkultur zugeschrleben. Ob das der Wirklichkeit entspricht oder nicht werden wir versuchen zu verdeutlichen.

II.3.3. D. J. Telegin ist der Meinung, daß "die meisten Forscher...den Steppeneinfluß als Prozeß auf Südosteuropa als dauernd und mehrfach (verstehen, U. A. ). Dabel gilt die Tatsache der Verbreitung auf den Balkan und ins Donaugebiet von Hügelgräbern der Grubengrabkultur des späten Äneolithikums als allerweiskräftigste"44. Nach demselben Autor wird die Grubengrabkultur "jetzt ganz bestimmt zur Periode vom XOVII. bis zum XIX. Jh. v.u.Z. datiert'145.

Der westlichste Widerhall dieses Vordringens ist in Serbien zu verzeichnen. Wahrscheinlich führte diese Bewegung im südlichen Banat zur Unterbrechen der Entwicklung der Kostolac-Kultur ${ }^{46}$ und sehr möglich zu elner "Okkupation" durch die Steppenvölker" ${ }^{47}$ Das wäre ungefähr um 2300-2200 v. Chr., also in elner "Zeit nach der Cotofeni-Kultur in Rumănien, die im Westen der Blüte der Vu²edol-Kultur entspricht"40.

Diese Datlerung ist im Einklang mit den C-14 Daten von Padej ${ }^{49}$ und Kétegyháza (Greb 4 aus dem Tumulus 3$)^{50}$ und zeigt daß "the appearance of barrows whose deposit contains Baden, Kostolac, or Cotofeni pottery is a terminus post quem for tha Pit-grave culture sites in Romania,

\footnotetext{
3 Dergacev 1989, 136.

39 Dergacev (1994, 137) Ist der Melnung, daß die "Edinet-Kultur auf dem Gebiet der Moldau in Folge einer Migration elnes Teils der Bevolkerung der nord-bstlchen Regionen Ungams (Hatvan-Kultur !; U.A.)" erschienen ist. Dlese Hypothese haben wir schon In einem anderen Aufsatz in Frage gestellt und auf die Unmöglichkeit elnes Synchronlsmuses GlinaEdinet-Hatvan hingewiesen (Schuster 1998, 28).

to Über dle Edinet-Kultur: Dergacev 1986, 111 ff.; Dergacev 1994, 129, 137.

41 Dergacev 1986, 82-87; Dergacev 1994, 123-127; Bogataja, Manzura 1994, 81-85; Manzura, Sava 1994, $175-178$.

B Bogataja, Manzura 1994, Abb. $10 / 7$.

13 Manzura, Sava 1994, Abb. $10 / 7$.

4 Telegin 1987, 37.

45 Telegin 1987, 37.

4 Tasic 1994, 30.

47 Tasic 1994, 31, 33. Miluth Garasłanin unterstrelcht, daB sich volkerbewegungen in der Belotic-Bela Crkva-Zeit auch In Westserblen und Cetna, zwischen Cetina und Dalmaten und Nordalbanien abrollen (Garałanin 1994, 18).

Tasic 1994, 31.

- Giric 1982, 103; Tasic 1995, 74.

50 Escedy 1979, 52; Taslc 1995, 74.
} 
Hungary and Serbia" und entspricht desgleichen der entwickeltesten Stufe der Grubengrabkultur aus dem Dniepr-Dnestr-Gebiet.' ${ }^{\text {. }}$.

Die Steppengruppen gelangten nach Serbien und weiter bis zur Ostküste der Adria auf zwei Hauptwegen. Der erste führte über die Obere Theiß und der zweite entlang der Donau ${ }^{52}$. Dieses Vorstoßen ist an die späteste Steppenwelle anzuknüpfen.

II.3.4. Von Interesse für uns ist diese letzte Marschroute, denn sie erlaubten uns einiges mehr über den Drang nach Westen einiger Glina-Gemeinschaften zu veranschaulichen und zu verstehen.

In der Walachei und in Oltenien wurden nicht weit entfernt von der Donau, aber auch im Innenland, mehrere Gräber entdeckt, die den aus dem Osten kommenden Steppenvölker zugeordnet werden können. Die Funde im nördlichen Teil Munteniens bei Ploiești-Triaj ${ }^{53}$ (einige der Gräber), und die in den :mittleren und westlichen Zonen bei Sănouliţa-Săruleşti ${ }^{54}$, Sultana ${ }^{55}$, Pneasna-Gurbănești ${ }^{56}$,

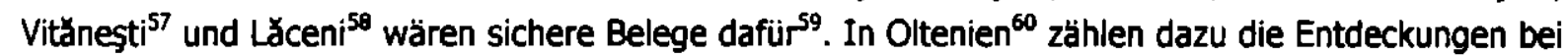
Rusăneşti, Rast, Seaca de Câmpie, Basarabi, Giubegea-Perişor, Hunia, Pleniţa und Suharu-Dolj. Dieser letzte Fund ${ }^{61}$ wurde von Florin Burtănescu als ein Beweis einer nicht-Jamna-Geineinschaft, die aber auch aus dem Osten gekommen ist, gedeutet ${ }^{62}$.

Eine weitere interessante Entdeckung in Oltenien sind die Gräber aus den Tumuli bei Verbitga ${ }^{63}$. Diese gehören gemräß den meisten Forschern der Glina-Kultur, ihrer lezten Stufe (IV. nach Roman), $a n^{64}$. Trotz dessen stellen wir uns bei einer eingehender Analyse die Frage, ob auch hier nicht Elemente der Jamna-Kultur zu finden sind und eine neue Zuordnung der Tumuli nötig ist ${ }^{65}$.

Von éen erwähnten Funden, liegen an der Donau (oder unweit gelegen von ibr) die Gräber von Vlădeni-Cătănoiu, Grădiştea-Coslogeni, Sultana, Rusănesti, Rast, Basarabi. Die anderen liegen im Innenland. Diesbezüglich bevorzugten die Nomaden-Gemeinschaften in dieser Periode in der Walachei das Mostiştea-Tał (Sănduliţa-Sarulești, Preasna-Gurbăneşti und Sultana) und das Teleorman-Becken (Vităneşti, Lăceni). Das heißt oder sehr weit entfernt von dem Glina-Verbreitungsgebiet, wie im Falle des Mittleren Ialomiţa-Laufs, oder an seiner östlichen Grenze, das Mostiştea-Tal.

Die zwei Funde am Teleorman-Fluß bestätigen erneut, daß die Glina-Gemeinschafteo dieses in der Nähe der Donau liegenden Gebiet nicht konntroliert haben. Aber es erklärt auch, warum die Glina-Siedlung von Orbeasca de Sus befestigt wurde ${ }^{66}$.

Alle Jamna-Spuren aus der Walachei und Oltenien gehören der letzten Welle an. Eine Ausnahme wäre vielleicht der Fund von Vitaneşti. Das könnte eine mögliche Erklärung für die

\footnotetext{
S1 Tasic 1995, 74.

\. Srejovic 1987, 49 und Karte 2. S. auch Rorin Medeleţ und Ioan Bugilan (1989) für das "rumthische" Banat

5 Netor 1944, 29-30; Nestor 1944a, 55-56; Comşa 1989, 181-188; Roman et all. 1992, $104-105$.

s Ulanici 1984, 113-116.

55 Morint, Ionescu 1968, 114118. Weitere Informationen gab uns Herr Done Serbănescu und Fräulein Alecandra Comșa, denen wir dafür aus ganzen Herzen danken.

$\$$ Rosetti 1959, 791-812; Roman et all. 1992, 104.

57 Leahu, Trohani 1979, 128, 133; Leahu, Trohani 1986, 21-26.

Stola 1981, 371.

99 Es wurden auch Bestattungen gefunden, deren Datlerung und kulturelle Zuordnung insicher ist.

o Odobescu 1878, 64; Nicoläescu-Plopsor 1923a, 81-84; Nlcolbescu-Plopsor 1923b, 85-87; Dumiltescu 1944a, 38-43; Dumitrescu 1944b, 84-87; Berclu et all. 1951, 228-245; Nicolăescu-Plopşor, Dumitrescu 1951, 275-277; Leahu, Trohan 1979, 127-141; Leahu, Trohant 1986, 21-26.

${ }_{61}$ Nicolăescu-Plopșor 1932.

Q Burtănescu 1996, 92.

${ }^{63}$ Berciu, Roman 1984, 15-21; Roman et all. 1992, 115-116;

S. auch Schuster 1997, 148.

${ }_{65}$ Mllutın Garašanln (1982, 170 sqq.; 1983, 463 sqq.; 1987, 33) schllebt dle Gräber von Vertita einem donauländlschbalkanischen Komplex eln.

\$6 Moscalu-Beda 1979, 368-369; Schuster 1997, 201.
} 
Wehranlagen der Glina-Siedlung bei Orbeasca de Sus, eine Niederlassung der klassischen Periode, sein.

Nicht zu vergeßen ist auch die Tatsache, daß klare Kontakte zwischen der Glina- und der Jamna-Kultur erst in der letzten Stufe (die IV. = Ostrovul Corbului nach Roman) der ersterwähnten Erscheinung zu sehen sind und das insbesondere in Oltenien. Aber diese Feststellung ist scheinbar im Gegensatz mit der Hypothese dernach ein Teil der Glina-Gemeinschaften von einer aus dem Osten vordringenden Steppenwelle in Bewegung gesetzt wurde.

Heute wird es immer klarer das nicht unbedingt eine große Nomadenpendelung die Hauptursache der nach Westen ziehenden Glina-Gruppen war, sondern daß es sich um eine Ansammlung von melireren Faktoren es sich handelt, unter denen auch das Steppcrelement seine Rolle spieite. Diese war in der ersten Stufe (II. nach Roman) der klassischen Periode (nach Schuster) gemäß dem jetzigen Forschungsstand noch minder, wuchs aber in der zweiten Stufe (III. nach Roman) immer mehr und erst in der letzten Stufe kommt es zu regelreckten festen kulturellen und ethnischen (?) Kontakten, so wie uns die Funde aus Oltenien aber auch aus Norwestbulgarien beweisen. Diesbezüglich schreibt Stefan Alexandrov neulich folgendes: "The tumuli from Hârlec, Tărnava (gr. NN 7-10), Love², indicate possible elements of "Late Pit Grave" type. Without entering into a detaileci discussior an the cultural interpretation of the Odeia Turcului and Naeni materials...we can postulate the existence of the Late Glina culture materials south of the Danube somewhere between the Balkan mountains and the Danube at the end of the EBA... The Glina III penetration north of the Danube as well as into the former Cotgofeni Culture regions south of the Danube is connected with tumulus graves (Harlec etc.) ${ }^{167}$.

Daß der Steppendruck in dem zweiten Teil der klassischen Periode der Gline-Kultur wuchs und daß einige Nomadengruppen versucht haben in den von ihr kontrollierten Raum einzudringen ist möglicherweise auch durch die Wehrgraben von Crivăţ ${ }^{60}$ und Odaia Turcului ${ }^{69}$ bekräftigt. Die letatgenannte Siedlung 'gehört der Glina III-Stufe ah, während jene von Crivat bis zu neuen Forschungen cier II. zugeordnet werden muß. Das hieißt, ocier daß es mehrere zeitverschiedene Elndringversuche der Nomaden gab, oder daß, wie wir schon unterstrichen haben ${ }^{70}$, die aus dem Westen entlang des oltenischein Hügellandes und weiter nach Südesten dem Argeş-Lauf entlang kommenden $\mathrm{Vu}^{2}$ edol-Einflüße, nicht allen Glina-Gemeinscinaften aus Muntenien eigen weren und dlese letzterwăhnten trotzdessen auf dieselbe Zeitstufe zu setzen sind.

Die Glina-Siedlufgen, il denen vu²edolbeeinflußte Keramik entdeckt wurde, zeigen uns den "Weg" den diese Einfüße zurückgelegt haben, aber auch, daß das in der Nähe der Donau gelegene Gebiet vermieden wurde, möglicherweise da dieses eben von den Steppenvölkerschaften in Besitz genommen wurde. Diese Hypothese könnte ihre Wiederspiegelung in der Wirklichkeit finden, wenn wir an die Konzentration der Jamna-Funde in der Băilesti-Ebene und seine Nachbargebiete (Rast, Seaca de Câmpie, Basarabi, Giubegea-Perişor, Hunia, Pleniţa und Suharu-Dolj) denken.

II.3.5. Anhand des oben vorgelegten, müßen wir schlußfolgern, daß die Steppenvölkerschaften den Raum östlich der Mostiştea während der ganzen Entwicklung der GlinaKultur durchstreifen, vielleicht sogar kontrolliert haben, und das obwohl dieses Gebiet relativ

\footnotetext{
${ }^{67}$ Alexandrov 1998, 230.

Berciu 1966, 534535; Schuster 1997, 190.

69 Tudor 1982, 60; Tudor 1983, 108. Wir sprachen (Schuster 1997, 30-31) anhand alteren Informationen auch von einigen Wehranlagen in Popeşti (Stadt Mihaileșt, Bezirk Giurgiu). Die neusten Forschungen wiedersprachen dieser Hypothese (Palincaş 1996, 239-288; Palincaş 1997, 175-182).

"Schuster 1997, 87.
} 
umweltunfreundlich war"1. Der Druck der Jamna-Gemeinschaften auf die Zonen, die von den GlinaStämmen bewohnt waren, lief ununterbrochen, hatte aber, wie scbon hervorgehoben, verschiedene Stärke, der maximale Punkt wird in der zweiten Stufe (Glina III) der klassischen Periode (nach Schoster) ereicht. Die Wege der zwei Kulturerscheinungen scheinen sich archäologisch erkennbar in der Glina IV-Stufe (= Ostrovul Corbului) zu "kreuzen" als beide sich in Westoltenien, im Donau-Gebiet möglich unsanft aufeinanderprallten. So kommt es, daß Glina-Keramik in den Gräbern der Grubengrabkultur erscheint und ern Tell der "verseuchten" Steppengemeinschaften nach Süden, über die Donau, schwenken und einige der Cotofeni-Gebiete in Nordwestbulgarien in Kraft nehmen.

II.3.6. Über die Rolle der Steppenvölker in dem Verschwinden der Glina-Kultur und das Auftreten neuer Kulturerscheinungerı wissen wir eigentlich wenig. Wenn die Glina-Kultur synchron mit der klassischen, möglich auch mit dem Anfang dar Endjamna-Kultur ist, so müßen die nach-Glina Äußerungen auf dieselbe Zeittreppe mit der Endperiode der letzten Erscheinung gesetzt werden. Interessant ist, daß sowohl die Odaia Turcului- wie auch die Năeni-Gruppe eher südliche als Steppeneinflüße aufweise ${ }^{72}$, während die Kistengräber aus der Nordwalachei ${ }^{73}$ einem breiteren europäischen Horizont, der dem Westbalkan (Cetina, Glasinac, Belotic-Bela Crkva) und Siebenbürgen (Livezile, Copăceni) eigen ist, angehören.

Der Druck der östlichen Nomaden scheint gegen das Ende der Frühbronzezeit (FB III nach Roman) Oltenien abzuflauen. Das erklärt auch warum das Gebiet zwischen dem Eisernen Tor und dem Alt allmählich von den aus dem Westen vorstoßenden Gornea-Orlești-Gemeinschaften besetzt wurde. Spuren dieser wurden in Ostrovul Corbului, Orlesti, Rogova, Ocnele Mari, Govora Sat entdeckt ${ }^{74}$.

In Muntenien sind bis jetzt keine klaren Funde dieses Typs zu verzeichnen ${ }^{75}$, obwohl angenommen wird daß dieser Kulturhorizont eine der Grundlagen für das Erscheinen der Tei-Kultur ist ${ }^{76}$.

\section{LITERATUR}

Alexandrov $1998=$ St. Alexandrov, Pottery from the End of the Early Bronze Age in West Bulgaria, in James Harvey Gaul in Memoriam. In the Steps of James Harvey Gaul, 1, Sofia, 223-233.

Berciu 1934 = D. Berciu, Materiale pentru preistoria Olteniei. II. Civilizatia de tip Glina III, Memoriul XXI al Institutului de Arheologie Olteană, Craiova, 14 S., 3 Abb., 1 Karte.

Berciu 1939 = D. Berciu, Arheologia preistorică a Olteniei, AO, 18, 104-106.

Berciu 1966 = D. Berciu, Rezultatele primelor săpături de la Crivăt (1965) (r. Olteniţa), SCIV, 17, 3, 529-535.

Berciu et all. 1951 = D. Berciu, E. Comşa, G. Marin, S. Morintz, C. S. Nicolăescu-Plopşor, S. PopescuIalomiţa, C. Preda, Şantierul arheologic Verbicioara Dolj, SCIV, II, 1, 228-245.

\footnotetext{
71 Schuster 1994a, 65-66; Schuster 1997, 83. Der östliche Teil der Walachei wird von seBhaften Gemeinschaften bis in die Mittelbronzezeit (Tei III) vermieden (Schuster 1994a, 171-178).

7 Schuster 1997, 124125.

${ }^{7}$ Schuster 1997, 126-131.

${ }^{4}$ Petre-Govora 1976, 16-17; Petre-Govora 1988, 137-146; Petre-Govora 1995, 41; Roman 1985, 120; Roman 1988, 218, 222; Crăciunescu 1997.

75 Schuster 1997, 161.

${ }^{7}$ Roman 1986, 31.
} 
Berciu, Roman 1984 = D. Berciu, P. Roman, Mormintele tumulare de la Verbita (jud. Dolj), ThracoDacica, V, 1-2, 15-21.

Bogataia, Manzura 1994 = L. K. Bogataia, I. Manzura, Ost-Westr-Wechselbeziehungen im Spiege/ der äneolithisch-frühbron-zezeitlichen Kulturen des nordwestlichen Schwarzmeergebietes, ZfA, 28-2, 63-86.

Burtănescu 1996 = F. Burtănescu, Consideraţii asupra unor morminte tumulare de pe teritoriul Moldovei (Periaada de tranzitie - Bronz timpuriu), Thraco-Dacica, XVII, 1-2, 87-116.

Comşa 1989

= E. Comşa, Mormintele cu ocru din movila II - 1943 de la Ploieşti-Triaj, Thraco-Dacica, X, 1-2, 181-188.

Dergacev 1986

= V. Dergacev, Moldavija I sosdenie territorii v epochu bronzy, Kišinev.

Dergacev 1989

= V. Dergacev, Zum Problem des Vordringens ästlichr Stämme in den karpatischen Donauraum vom Äneolithikum bis in die ältere Bronzezeit, in Das Äneolithikum und die früheste Bronzezeit (C 14 3000-2000 b.c.) in Mitteleuropa: kuthrelle umd chronologische Beziehungen (Acto des XIV. Internationalen Symposiums PragLiblice 20.-24.10.1986), Praehistorica, XV, 133-137.

Dergacev $1994=$ V. Dergacev, Epoca bronzu/ui. Prima periaada, Thraco-Dacica, XV, 1-2, 121-140.

Dumitrescu 1944a = V. Dumitrescu, RaportMNA, 38-43.

Dumitrescu 1944b = V. Dumitrescu, RaportMNA, 84-87.

Ecsedy 1979

Garašanin 1994

$=\mathrm{I}$. Ecsedy, The People of the Pit-Grave Kurgans in Eastem Hungary, Budapest.

= M. Garašanin, Pastoralisme semi-nomade et nomade dans la Peninsule Balkanique à l'eneolithique et du debut de l'Âge du bronze, Balcanica, XVV-1, 7-18.

Giric 1882 = Über die Erforschung der Grabhügel in der Wojwodina, Simposio LaziseVerona, Verona.

Leahu, Trohani 1979 = V. Leahu, G. Trohani, Săpăturile anheologice de la Vităneşti, CA, III, 127-141.

Leahu, Trohani 1986 = V. Leahu, G. Trohani, Săparturile arheologice efectuate in 1983 la Vităneşti, jud. Teleorman, CA, VIII, 21-26.

Machnik $1991=$ J. Machnik, The Earliest Bronze Age in the Carpathian Basin, Archaeological Sciences Bradford, Bradford.

Manzura, Sava 1994 = I. Manzura, E. Sava, Interacţiuni "est-vest" reflectate în culturile eneolitice șI ale epocii bronzului din zona de nord-vest a Marii Negre (Schita cultura/istorică), MemAnt, XIX, 143-192.

Medelet, Bugilan 1989 = F. Medelet, I. Bugilan, Contributii la problema şi la repertoriul movilelor de pămînt din Banat, Banatica, 9, 88-198.

Morintz, Ionescu 1968 = S. Morintz, B. Ionescu, Cercetări arheologice în împrejurimile Oltenitei (1958-1967), SCIV, 19, 1, 99-121.

Moscalu-Beda 1979 = E. Moscalu und C. Beda, Noi cetăti traco-getice, CA, III, 368-369.

Nestor $1960=$ I. Nestor, Epoca bronzului, in Istoria Romániei, I, Bucureşti, 90-132.

Nicolăescu-Plopşor 1923a = C. S. Nicolăescu-Plopşor, Măgura Mare din pădurea Poenifi-Plenita Dolj, Oltenia, culegeri-cercetări-documente, I, fasc. VI, 81-84.

Nicolăescu-Plopşor 1923b = C. S. Nicolăescu-Plopşor, Măgura din viea lvi Ion St. Bârţan-Plenita Dolj, Oltenia, culegeri-cercetări-documente, I, fasc. VI, 85-87.

Nicolăescu-Plopşor 1932 = C. S. Nicolăescu-Plopşor, Institutul de Arheologie Olteană. Memoriul VII, Craiova. 
Nicolǎescu-Plopşor, Dumitrescu 1951 = C. S. Nicolžescu-Plopşor, Vl. Dumitrescu, N. Gostar, Raport asupra activităţii şantierului arheologic Rast-Dolj. I. Săpăturile de la Grindul Şifarului şi II. Cimitirul tumular de pe Islazul comunelor Rast şi Seaca de Câmpie, SCIV, II, 1, 267-277.

Odobescu 1878

Palincaş 1996

Palincaş 1997

Petre-Govora 1976

Petre-Govora 1988

Roman 1976

Roman 1985

Roman 1985a

Roman 1986

Roman 1988

Roman 1996

Roman et all. 1992

Rosetti 1959

Schuster 1994

Schuster 1994 a

Schuster 1997

Schuster 1997a

Schuster 1998

Schuster 1998a

Srejovic 1987

Stoia 1981

Zs. Székely,
= Al. Odobescu, Antichitătile judetului Romanaţi, București.

$=\mathrm{N}$. Palincas, Valorificarea arheologiç a probelor $14 C$ din fortificatia aparţinând Bronzului târziu de la Popesti (jud. Giurgiu), SCIVA, 47, 3, 239-288.

= N. Palincaş, Scurtă prezentare a săpăturilor din sectorul $\Sigma$ al aşezării de la Popesti (jud. Giurgiu). Campaniile 1988-1993, CA, X, 173-192.

= Gh. Petre-Govora, Aspecte ale inceputului epocii bronzului in nord-estul Olteniei, Buridava, 2, 7-33.

$=\mathrm{Gh}$. Petre-Govora, Descoperiri arheologice din O/tenia privind epoca timpurie a bronzului, Thraco-Dacica, IX, 1-2, 137-147.

= P. Roman, Die Glina III-Kultur, PZ, 51, 1, 26-42.

= P. Roman, Perioada timpurie a epocii bronzului tracic in Oltenia, ThracoDacica, VI, 1-2, 116-122.

= P. Roman, Cercetări la Govora Sat-Runcuri în 1977, SCIVA, 36, 4, $279-297$.

= P. Roman, Perioada timpurie a epocii bronzului pe teritoriul Românie, SCIVA, 37, 1, 29-55.

= P. Roman, Ostrovul Corbului (Rumänien) und Malé Kosihy (Tschechoslowakei), SlovArch, 36, 217-224.

= P. Roman, Ostrovul Corbului. Istoricul cercetarrii. Săpăturile arheologice şi stratigrafie, I, 1a, 1-33 (in Deutsch: 35-65).

= P. Roman, A. Dodd-Opritescu, P. János, Beiträge zur Problematik der schnurverzierten Keramik Südosteuropas, Heidelberger Akademie der Wissenschaften. Monographien III, Mainz am Rhein.

= D. V. Rosetti, Movile funerare de la Gurbănesti, MCA, VI, 791-812.

= C. Schuster, Despre aria de răspándire a culturii Glina, Istros, VII, 63-70.

= C. Schuster, Aria de răspândire a culturii Tei, AB, 5.N., III, 171-178.

= C. Schuster, Perioada timpurie a epocii bronzului în bazinele Argeşului şi Ialomitei Superioare, Bibliotheca Thracologica, XX.

= C. Schuster, Despre locuintele culturii Glina, MemAnt, XXI, 85-104.

$=\mathrm{C}$. Schuster, Die Frühbronzezeit in Südostsiebenbürgen und in der Walachei Verbindungen, Beziehungen, kulturelle Einflüsse, AMN, 35, 1, 25-35.

= C. Schuster, Sur le "mouvement" dans le Bronze roumain, Bulletin de Thracologie, IV, 187-191.

= D. Srejovic, Die Hauptwege des Vorstosses der Steppenkutturen auf den Balkan, in Hügelbestattungen in der Karpaten-Donau-Balkan-Zone, Beograd, 45-49.

= A. Stoia, Les fouilles archéologiques en Roumanie (1980), Dacia, N.S., XOV, 363-379.

= Perioada timpurie şi începutul celei mijlocii a epocii bronzului în sud-estul Transilvaniei, Bibliotheca Thracologica, XXI, Bucuresti.

Şerbănescu, Trohani 1978 = D. Serbănescu, G. Trohani, Cercetările arheologice de pe Valea Mostiştei, in Ilfov. File de Istorie, Bucureşti, 18-32. 
Tasic 1994

Tasic 1995

Telegin 1987

Tudor 1982

Tudor 1983

Ulanici 1979

Ulanici 1984

Vulpe 1990
$=\mathrm{N}$. Tasic, Das archäologisch-historische Bild der Entwicklung der Kulturen des Äneolithikums im südlichen Banat, Balcanica, XXV-1, 19-37.

= N. Tasic, Eneolithic cultures of Central and West Balkans, Belgrade.

= D. J. Telegin, Über kulturelle Kontakte zwischen der neo-äneolithischen Bevölkerung des nordpontischen Gebietes und der Balkan-Donauregion, in Hügelbestattungen in der Karpaten-Donau-Balkanzone, Beograd, 37-44.

= E. Tudor, Neue Angaben zur frühen Bronzezeit in Südrumänien, Dacia, N.S., XXVI, 59-75.

= E. Tudor, Săpăturile arheologice de la Odaia Turcului (jud. Dîmbovita), MCA, Braşov, 108-111.

= A. Ulanici, Srăturile arheologice efectuate la Branet in anul 1976, CA, III, 27-38.

= A. Ulanici, Săpăturile arheologice de la Săndulita, com. Săruleşti, jud. Călăraşi, CA, VII, 113-116.

= A. Vulpe, Neue Beiträge zur Chronologie und kulturellen Gliederung der Frühbronzezeit im unteren Donaugebiet, Starinar, XL-XLI, 105-111.

\section{ABKÜRZUNGEN}

$A B$

AMN

AO

Balcanica

Banatica
= Analele Banatului, Timişoara.

= Acta Musei Napocensis, Cluj-Napoca.

$=$ Arhivele Olteniei, Craiova.

= Balcanica, Beograd.

= Banatica, Resiţa.

Bulletin de Thracologie $=$ Bulletin de Thracologie, Institutul Român de Tracologie, Mangalia, București.
CA
= Cercetări Arheologice, Muzeul Naţional de Istorie a României, București.
Dacia
= Dacia, N.S., Institutul de Arheologie "V. Pârvan", București.
Istros
= Istros, Brăila.
MCA
$=$ Materiale şi cercetări arheologice.
Oltenia
= Oltenia, Craiova.
Praehistorica
$=$ Praehistorica, Praha.
$P Z$
= Prähistorische Zeitschrift, Berlin, Mainz.
SCIV
= Studii şi cercetări de istorie veche, Bucureşti.
SCIVA
= Studii şi cercetări de istorie veche și arheologie, București.
SlovArch
= Slocvenská Archeológia, Bratislava.
Starinar
= Starinar, Beograd.
SympThrac
= Symposia Thracologica, Institutul Român de Tracologie.
Thraco-Dacica ZfA
= Thraco-Dacica, Institutul Român de Tracologie, București.
$=$ Zeitschrift für Archäologie, Berlin. 